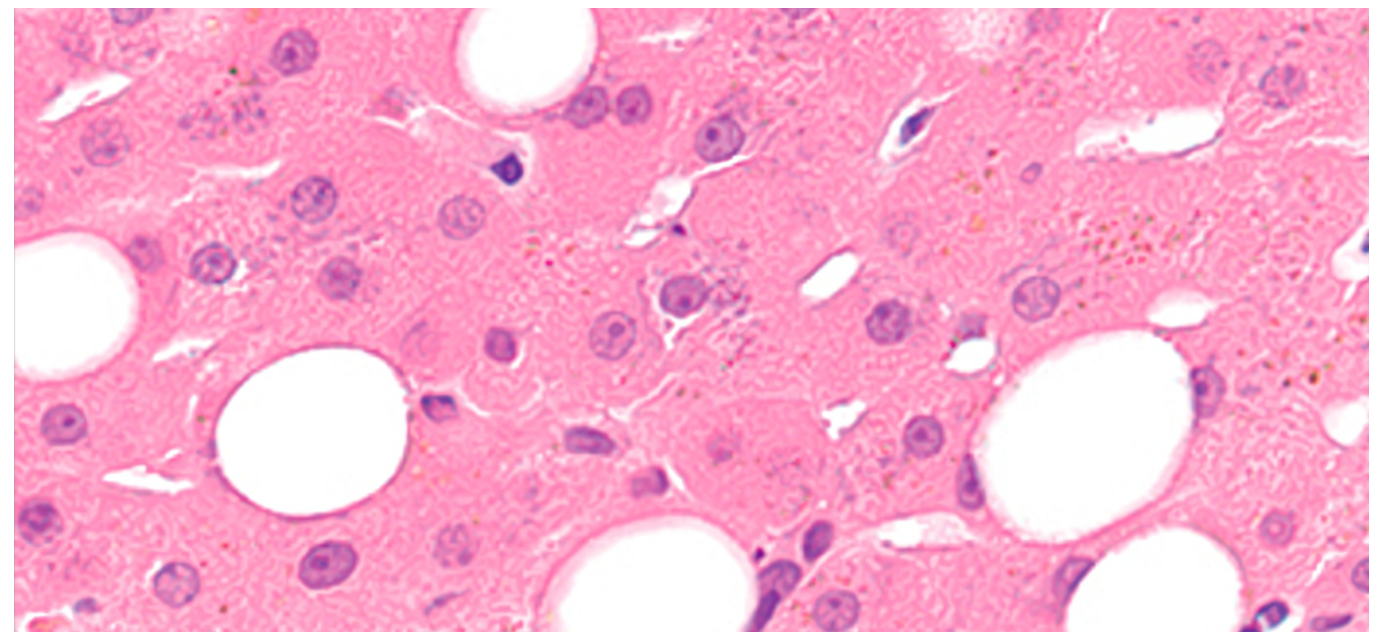

\title{
On the operational definition of fatty liver
}

\author{
Giorgio Bedogni ${ }^{1}$, Ferruccio Bonino ${ }^{2}$, Claudio T iribelli \\ 1 Fondazione Italiana Fegato ONLUS \\ 2 University of Pisa
}

Abstract

We discuss the present operational definition of fatty liver and its shortcomings.

\section{Definitions}

Fatty liver (liver steatosis)

Defined by EASL-EASD-EASO

True positive fraction

Defined by Margaret Pepe

True negative fraction

Defined by Marg aret Pepe

Diagnostic likelihood ratio

Defined by Marg aret Pepe

Non-alcoholic fatty liver disease (NAFLD)

Defined by EASL-EASD-EASO

Non-alcoholic steatohepatitis (NASH)

Defined by EASL-EASD-EASO

On the diagnosis of fatty liver 
Fatty liver or liver steatosis is presently defined as an intrahepatic triglyceride content > $5 \%{ }^{[1]}$. A strict diagnosis of fatty liver requires liver biopsy, which is presently used to classify steatosis as light (> $5 \%$ to $\leq 33 \%$ ), moderate (> $33 \%$ to $\leq 66 \%$ ) or severe (> $66 \%)^{[2]}$.

Although liver biopsy is the reference method for the diagnosis of fatty liver, it is an imperfect gold-standard because of sampling error. A needle biopsy is in fact approximately $1 / 20000^{\text {th }}$ of the weight of the liver ${ }^{[2]}$ and even if the most common intrahepatic fat pattern is the diffuse form, there are also heterogeneous, focal, multinodular, perilesional, perivascular, subcapsular, and lobar forms ${ }^{[3]}$.

In addition, liver biopsy is an invasive procedure and cannot be employed outside tertiary care centers and even in these centers it is nearly always performed on selected patients ${ }^{[4]}$. Importantly, liver biopsy is also the reference method for diagnosis of both intrahepatic inflammation and liver fibrosis, as we shall see below.

Proton magnetic resonance spectroscopy or quantitative fat/water selective magnetic resonance imaging (MRI) can detect an intrahepatic triglyceride content > 5\% (corresponding to a proton density fat fraction > 5.6\%) and could thus be used to diagnose fatty liver ${ }^{[1]}$. However, proton magnetic resonance spectroscopy is available only in few centers and mostly for research purposes. In addition, there is not a linear association between $5 \%$ fatty hepatocytes and $5 \%$ of whole liver weight fat content ${ }^{[5]}$.

Liver ultrasonography is the imaging method most commonly employed to diagnose fatty liver in the general population and in clinical practice ${ }^{[1]}$. When compared with liver biopsy, liver ultrasonog raphy has a true positive fraction of $85 \%$ (95\% confidence interval 80 to $89 \%$ ), a true negative fraction of $94 \%$ ( 88 to $97 \%$ ), a positive diagnostic likelihood ratio of 13.3 (6.4 to 27.6), and a negative diagnostic likelihood ratio of $0.16(0.12$ to 0.22$)$ for the detection of an intrahepatic triglyceride content $>33 \%{ }^{[6]}$. Degrees of fatty liver $<33 \%$ may go undetected by liver ultrasonography, especially when $<10 \%{ }^{[6]}$. A further option to study fatty liver in the general population is the use of surrogate markers $^{[1]}$. One of such markers, the fatty liver index (FLI), which we developed against 
liver ultrasonography in the general population of the Dionysos Nutrition \& Liver Study, is based on four common measurements, i.e. body mass index, waist circumference, gamma-glutamyl-transferase and triglycerides ${ }^{[7]}$. FLI has gained increasing attention in the last decade because of its association with prevalent and incident cardio-metabolic disease. More importantly for its ability to serve as surrogate marker of fatty liver, FLI has been successfully cross-validated in external populations ${ }^{[8]}$. Although the cut-points of FLI proposed by us to rule in and rule out fatty liver in the general population of Campogalliano (Modena, Italy) are commonly employed in the literature ${ }^{[7]}$, it is much better to recalibrate FLI in (a subsample of) the population of interest, as we advised in the original paper and as it was recently done by some researchers in the Shanxi Province of China ${ }^{[9][10]}$.

Liver biopsy remains the only method able to distinguish intrahepatic inflammation from fibrosis whereas liver elastometry and multiparametric magnetic resonance provide an indirect and combined measure of the two parameters, represented by two distinct vectors that are not yet distinguishable one from the other in both US and MRI-based imaging techniques ${ }^{[11][12][13][14]}$.

\section{On the dichotomization of fatty liver into alcoholic and non- alcoholic fatty liver}

Fatty liver is presently classified into non-alcoholic fatty liver disease (NAFLD) and alcoholic fatty liver disease (AFLD). The European Association for the Study of the Liver (EASL) presently suggests that, after (at least) hepatitis B infection, hepatitis C infection and steatogenic drugs have been ruled out, NAFLD should be diagnosed when ethanol intake is $\leq 20 \mathrm{~g} /$ day in women and $\leq 30 \mathrm{~g} /$ day in men (Fig ure 1). 


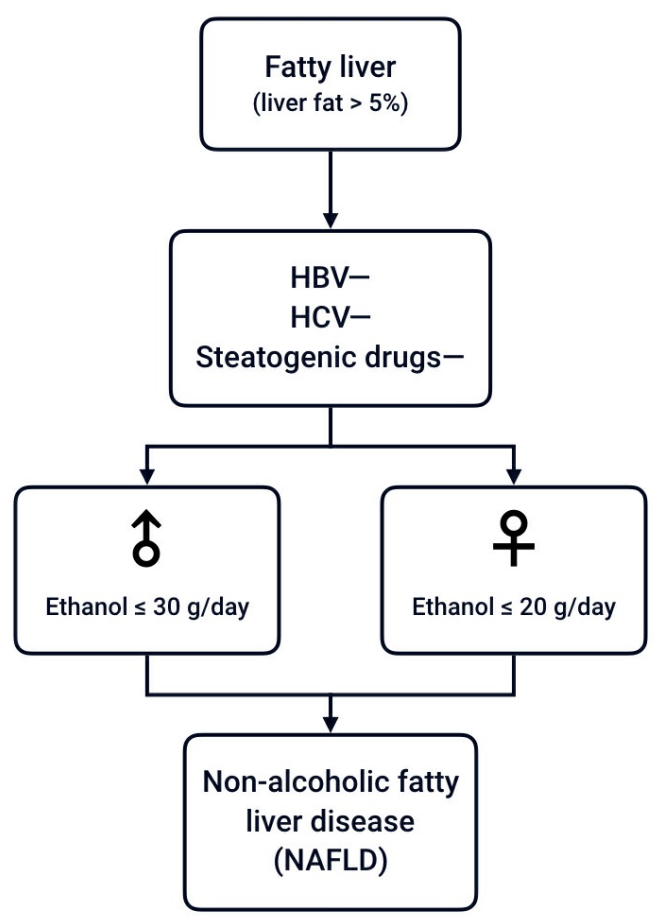

Figure 1 - The diagnosis of non-alcoholic fatty liver disease. Abbreviations: HBV = hepatitis B virus; HCV = hepatitis C virus; NAFLD = non-alcoholic fatty liver disease .

The NAFLD vs. AFLD dichotomization has several limitations.

Besides the loss of information inherent to any dichotomization ${ }^{[15]}$, its most important criticism is that a simple cut-off of low daily alcohol intake cannot really disting uish alcohol- and non-alcohol-induced liver damage. The toxicity from low alcohol intake is in fact determined by genetic, alimentary and lifestyle factors and some individuals may well have alcohol-induced fatty liver while drinking alcohol below the proposed cut-point ${ }^{[16]}$. On the other hand, the gut microbiota may produce alcohol contributing to liver damage $e^{[17]}$.

Another problem of the NAFLD vs. AFLD dichotomization is that it requires the use of an instrument accurate enough to detect small differences in ethanol intake ${ }^{[18]}$. Even the 7day weighed food record that we employed in the Dionysos Nutrition \& Liver study may not be accurate enough to detect small differences in ethanol intake ${ }^{[19]}$. To gain some understanding of the problems caused by dichotomization and/or measurement error, Figure 2 simulates the prevalence of NAFLD obtained by varying the cut-point of ethanol intake from 0 to $40 \mathrm{~g} /$ day by steps of $5 \mathrm{~g}$ /day in the general population 
investigated by the Dionysos Nutrition \& Liver Study ${ }^{[19]}$.

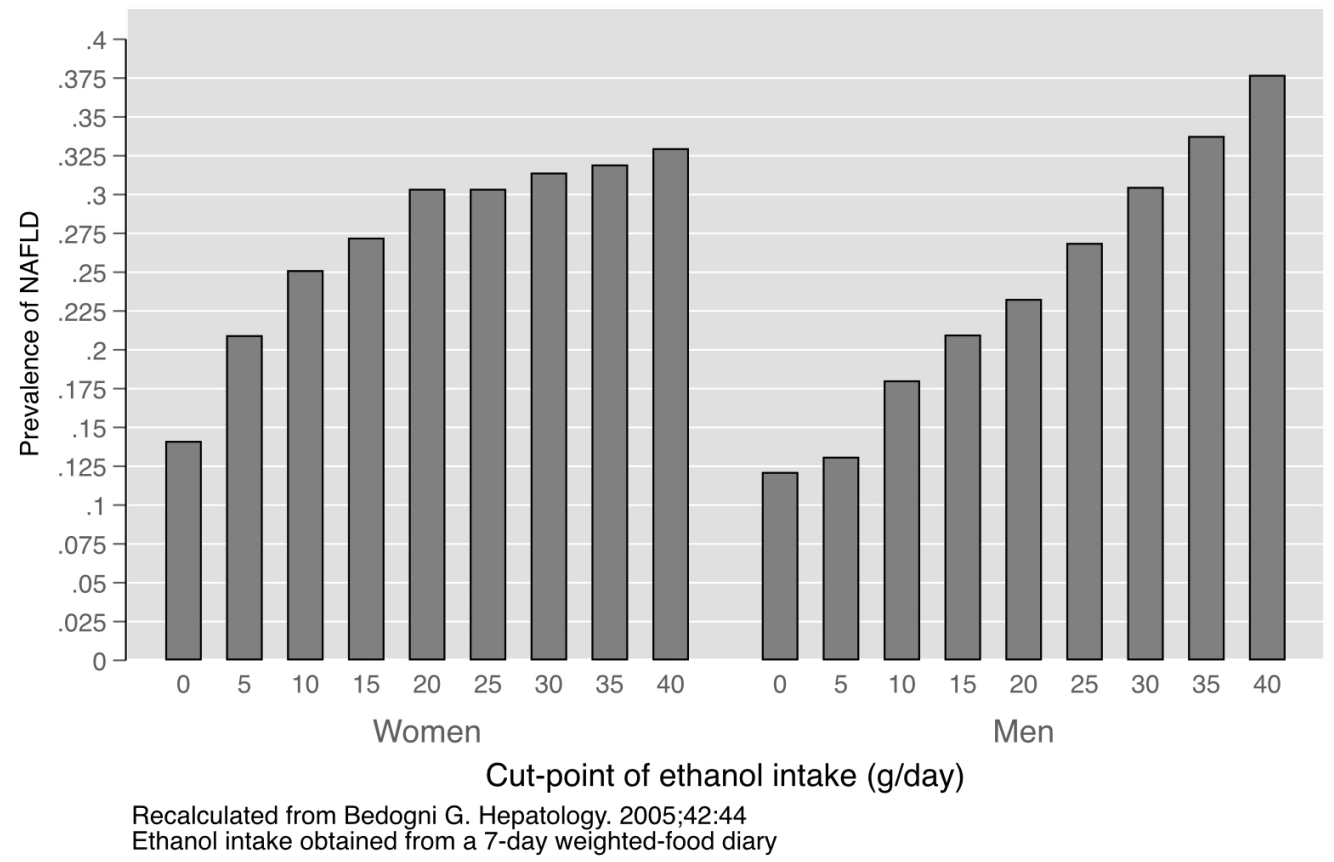

Figure 2 - Simulation of NAFLD prevalence obtained by varying the cut-point of ethanol intake from 0 to $40 \mathrm{~g} /$ day by steps of $5 \mathrm{~g} /$ day in the general population of the Dionysos Nutrition \& Liver Study.

At the time of the Dionysos Nutrition \& Liver Study, the cut-point of ethanol intake suggested by EASL to separate NAFLD from AFLD was $20 \mathrm{~g} /$ day for both men and women. If we adopt the current EASL cut-point of $30 \mathrm{~g} /$ day, the prevalence of NAFLD in the men of the Dionysos Nutrition \& Liver Study would be substantially higher. However, the (lack of) association between fatty liver and continuous ethanol intake that we have reported in the same population would obviously not chang $\mathrm{e}^{[7]}$. This alerts to a clear problem with dichotomization ${ }^{[15]}$.

\section{On the diagnosis of non-alcoholic steatohepatitis}

NAFLD is presently classified into uncomplicated non-alcoholic fatty liver (NAFL) and non-alcoholic steatohepatitis (NASH) ${ }^{[1]}$ (Fig ure 3). 


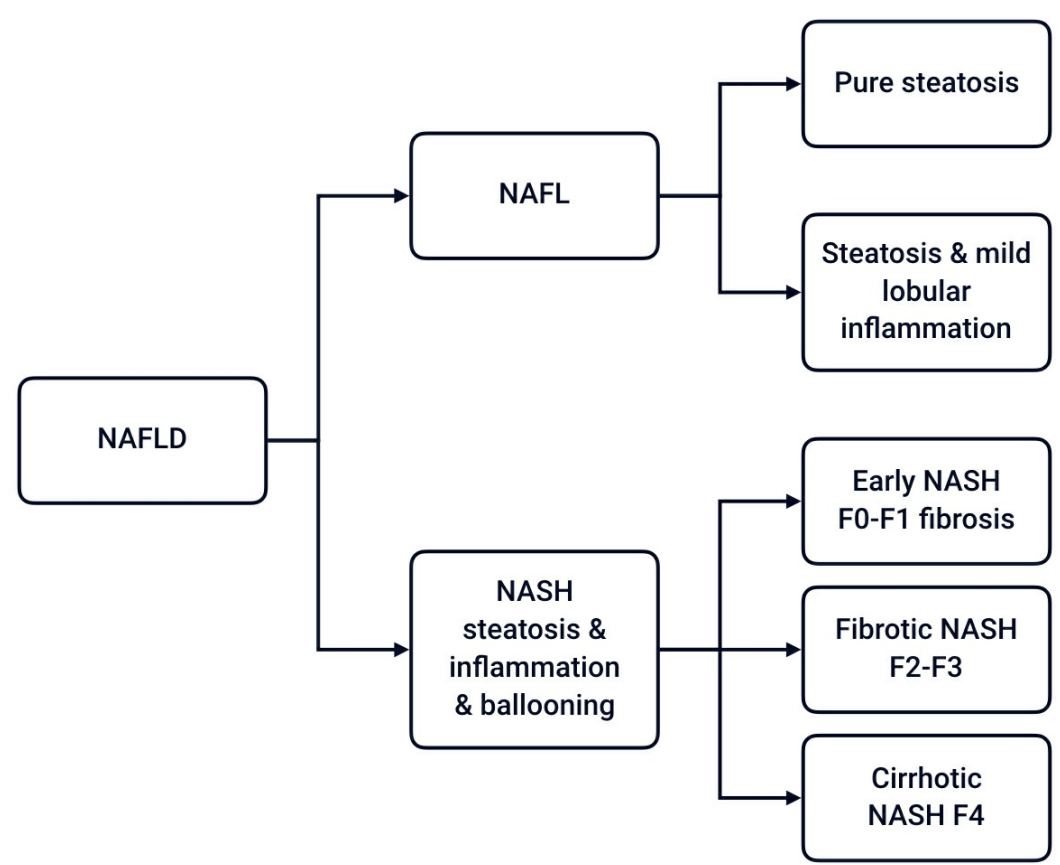

Figure 3 - Classification of non-alcoholic [fatty liver] disease into non-alcoholic fatty liver and nonalcoholic steatohepatitis. Abbreviations: NAFLD = non-alcoholic fatty liver disease; NAFL = non-alcoholic fatty liver; $\mathrm{NASH}=$ non-alcoholic steatohepatitis.

NASH is presently defined as the presence of steatosis, inflammation and ballooning at liver biopsy in a patient with NAFLD ${ }^{[1]}$. Liver biopsy cannot disting uish alcohol- from nonalcohol-induced steatohepatitis and the current separation of NASH and alcoholic steatohepatitis is based on the dichotomization of alcohol intake with the limitations described above. We refer the reader to a recent review for details and controversies on the histopathology of NASH ${ }^{[2]}$.

Here, we are interested only in the methodological consequences of the fact that liver biopsy is central to the diagnosis of steatohepatitis, independently of its etiology. This requirement implies that NASH cannot be diagnosed in the general population. Even if accurate indirect markers of NASH were available, which are not ${ }^{[1]}$, they would have been developed in tertiary care centers where liver biopsy can be performed, most often on selected subsets of patients ${ }^{[4]}$. Accordingly, their diagnostic performance is likely to be completely different in the general population because of the different case-mix of individuals $^{[20]}$. Thus, the available estimates of the prevalence of NASH in the general population should be taken with caution, especially when they are based on indirect and demonstrably unreliable markers of NASH such as altered liver enzymes in the presence 
of fatty liver after the exclusion of some of the known risk factors for this latter ${ }^{[21][22]}$.

\section{On the diagnosis of liver fibrosis}

The clinical relevance of NASH stems from its potential association with liver fibrosis ${ }^{[1]}$. Fig ure 4 shows the time to the progression of one stage of fibrosis for NAFL vs. NASH according to a recent metanalysis of long itudinal studies performed in tertiary care centers $^{[23]}$.

\begin{tabular}{|c|c|c|c|}
\hline & N & Studies & $\begin{array}{c}\text { Fibrosis progression } \\
\text { (years to progress 1 } \\
\text { stage, mean [95\%CI]) }\end{array}$ \\
\hline NAFLD & 366 & 11 & $7.7[5.5$ to 14.8$]$ \\
\hline NAFL & 133 & 6 & $14.3[9.1$ to 50.0$]$ \\
\hline NASH & 116 & 7 & $7.1[4.8$ to 14.3$]$ \\
\hline
\end{tabular}

Figure 4 - Time to the progression of one stage of fibrosis in non-alcoholic fatty liver disease. Abbreviations: NAFLD = non-alcoholic fatty liver disease; NAFL = non-alcoholic fatty liver; NASH = nonalcoholic steatohepatitis.

As stated above, only liver biopsy can distinguish inflammation from fibrosis and provide an accurate grading and staging of these two histologic features. Many surrogate markers and an increasing number of elastographic techniques are nonetheless available to provide a non-invasive measure of the combined vectors of inflammation and fibrosis without being able to discriminate among them ${ }^{[13][24][25]}$. As for NASH, these methods were calibrated against liver biopsy in tertiary care centers and most often on selected sets of patients so that their diagnostic performance is likely to be completely different in the general population because of the different case-mix of individuals ${ }^{[20]}$. Thus, the available estimates of the prevalence of NASH-associated liver fibrosis in the general population should be taken with caution ${ }^{[21][22]}$.

Following the Metavir system, liver fibrosis is currently classified in 5 stages: F0, F1, F2, F3 and F4, where F0 means absence of fibrosis and F4 is synonym with liver cirrhosis ${ }^{[2]}$. Instead of the common practice of dichotomizing fibrosis, much can be gained by 
treating it as an ordinal outcome and by developing predictive models giving cumulative probabilities $^{[26]}$. As an example of this application, Fig ure 5 gives the cumulative probability of the F0-F1 vs. F2-F3 vs. F4 stages of fibrosis as measured by Fibroscan among a series of Italian HCV patients ${ }^{[27]}$. According to the employed ordinal generalized logistic model, a patient with a liver stiffness of $12 \mathrm{kPa}$ would have a $2 \%$ (95\% Cl 0 to 5\%) probability of $\mathrm{F} 0-\mathrm{F} 1,85 \%$ (72 to $99 \%$ ) probability of $\mathrm{F} 2-\mathrm{F} 3$ and $13 \%$ (1 to $25 \%$ ) probability of F4 fibrosis.
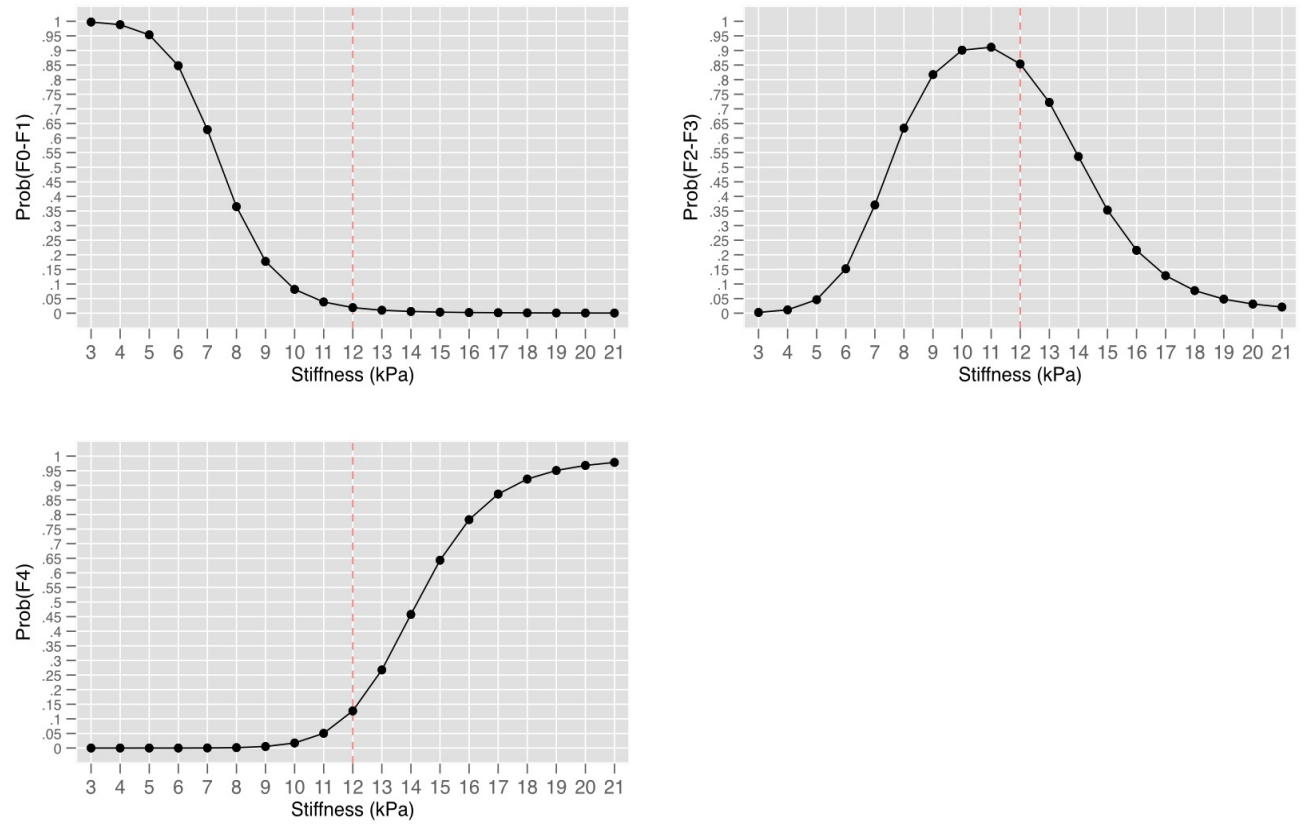

Recalculated from Arena et al. Hepatology 2013;58:65

Figure 5 - Cumulative probability of the F0-F1 vs. F2-F3 and F4 stages of liver fibrosis as predicted by an ordinal generalized logistic regression model (see reference ${ }^{[27]}$ for details). According to this model, a patient with a liver stiffness of $12 \mathrm{kPa}$ (red line) would have a $2 \%$ (95\% Cl 0 to 5\%) probability of F0-F1, $85 \%$ (72 to $99 \%$ ) probability of F2-F3, and $13 \%$ probability (1 to $25 \%$ ) of F4 fibrosis. Abbreviations: Prob. = probability.

\section{Conclusion}

We have described some limitations of the current operational definition of fatty liver and NAFLD. They can be summarized as follows:

1. Fatty liver is presently defined as an intrahepatic triglyceride content $>5 \%$ but the most commonly most used diagnostic technique, i.e. liver ultrasonog raphy, cannot 
reliably detect values of fatty liver $<10 \%$ and is much better at detecting values $>33 \%$. This is likely to cause an underestimation of fatty liver;

2. Instead of dichotomizing fatty liver into NALFD and AFLD, it would be more useful if epidemiological studies could evaluate the association between fatty liver and continuous or ordinal ethanol intake with the possibility of studying its independent contribution and interaction with other factors, e.g. body mass index;

3. The present estimates of the burden of NASH and its complications (fibrosis, cirrhosis, hepatocarcinoma) in the general populations are based on indirect assumptions and may not be reliable. The real burden of NASH and NASH-associated fibrosis in the general population is likely to remain unknown.

\section{References}

1. $\mathrm{a}, \mathrm{b}, \mathrm{c}, \mathrm{d}, \mathrm{e}, \mathrm{f}, \mathrm{g}, \mathrm{h}$. (2016). EASL-EASD-EASO Clinical Practice Guidelines for the management of non-alcoholic fatty liver disease Journal of Hepatology, vol. 64 (6), 1388-1402. doi:10.1016/j.jhep.2015.11.004

2. a, b, c, d David E. Kleiner, Hala R. Makhlouf. (2016). Histology of Nonalcoholic Fatty Liver Disease and Nonalcoholic Steatohepatitis in Adults and Children Clinics in Liver Disease, vol. 20 (2), 293-312. doi:10.1016/j.cld.2015.10.011

3. ^ Ilkay S. Idilman, Ilknur Ozdeniz, Musturay Karcaaltincaba. (2016). Hepatic Steatosis: Etiology, Patterns, and Quantification Seminars in Ultrasound, CT and MRI, vol. 37 (6), 501-510. doi:10.1053/j.sult.2016.08.003

4. a, b Nadege T. Gunn, Mitchell L. Shiffman. (2018). The Use of Liver Biopsy in Nonalcoholic Fatty Liver Disease Clinics in Liver Disease, vol. 22 (1), 109-119. doi:10.1016/j.cld.2017.08.006

5. ^ Thomas Karlas, David Petroff, Magali Sasso, Jian-Gao Fan, Yu-Qiang Mi, Victor de Lédinghen. (2017). Individual patient data meta-analysis of controlled attenuation parameter (CAP) technology for assessing steatosis Journal of Hepatology, vol. 66 (5), 1022-1030. doi:10.1016/j.jhep.2016.12.022

6. a, b Ruben Hernaez, Mariana Lazo, Susanne Bonekamp, Ihab Kamel, Frederick L. Brancati, Eliseo Guallar. (2011). Diagnostic accuracy and reliability of ultrasonography for the detection of fatty liver: A meta-analysis Hepatology, vol. 54 (3), 1082-1090. doi:10.1002/hep.24452

7. a, b, c Giorgio Bedogni, Stefano Bellentani, Lucia Miglioli, Flora Masutti, Marilena Passalacqua, Anna Castiglione. (2006). The Fatty Liver Index: a simple and accurate 
predictor of hepatic steatosis in the general population BMC Gastroenterol, vol. 6 (1) doi:10.1186/1471-230x-6-33

8. ^ Fatty liver index - references. http://citations.springer.com/item?doi=10.1186/1471$230 \times-6-33$

9. ^ Ewout W. Steyerberg. (2009). Clinical Prediction Models - A Practical Approach to Development, Validation, and Updating Springer

10. ^ Ya-Nan Shen, Ming-Xing Yu, Qian Gao, Yan-Yan Li, Jian-Jun Huang, Chen-Ming Sun. (2017). External validation of non-invasive prediction models for identifying ultrasonography-diagnosed fatty liver disease in a Chinese population Medicine, vol. 96 (30), e7610. doi:10.1097/md.0000000000007610

11. ^ B. Coco, F. Oliveri, A. M. Maina, P. Ciccorossi, R. Sacco, P. Colombatto. (2007). Transient elastography: a new surrogate marker of liver fibrosis influenced by major changes of transaminases J Viral Hepat, vol. 14 (5), 360-369. doi:10.1111/j.13652893.2006.00811.x

12. ^ Michael Pavlides, Rajarshi Banerjee, Joanne Sellwood, Catherine J. Kelly, Matthew D. Robson, Jonathan C. Booth. (2016). Multiparametric magnetic resonance imaging predicts clinical outcomes in patients with chronic liver disease Journal of Hepatology, vol. 64 (2), 308-315. doi:10.1016/j.jhep.2015.10.009

13. a, b Marcello Mancini, Paul Summers, Francesco Faita, Maurizia R Brunetto, Francesco Callea, Andrea De Nicola. (2018). Digital liver biopsy: Bio-imaging of fatty liver for translational and clinical research WJH, vol. 10 (2), 231-245. doi:10.4254/wjh.v10.i2.231

14. ^ Nicole Di Lascio, Cinzia Avigo, Antonio Salvati, Nicola Martini, Monica Ragucci, Serena Monti. (2018). Steato-Score: Non-Invasive Quantitative Assessment of Liver Fat by Ultrasound Imaging Ultrasound in Medicine \& Biology doi:10.1016/j.ultrasmedbio.2018.03.011

15. a, b Problems Caused by Categorizing Continuous Variables. http://biostat.mc.vanderbilt.edu/wiki/Main/CatContinuous

16. ^ Veeral H. Ajmera, Norah A. Terrault, Stephen A. Harrison. (2017). Is moderate alcohol use in nonalcoholic fatty liver disease good or bad? A critical review Hepatology, vol. 65 (6), 2090-2099. doi:10.1002/hep.29055

17. ^ Gabriele Capurso, Edith Lahner. (2017). The interaction between smoking, alcohol and the gut microbiome Best Practice \& Research Clinical Gastroenterology, vol. 31 (5), 579-588. doi:10.1016/j.bpg.2017.10.006

18. ^ Giorgio Bedogni, Valerio Nobili, Claudio Tiribelli. (2014). Epidemiology of fatty liver: an update WorldJ Gastroenterol., vol. 20(27): 9050-9054 
19. a, b Giorgio Bedogni, Lucia Miglioli, Flora Masutti, Claudio Tiribelli, Giulio Marchesini, Stefano Bellentani. (2005). Prevalence of and risk factors for nonalcoholic fatty liver disease: The Dionysos nutrition and liver study Hepatology, vol. 42 (1), 44-52. doi:10.1002/hep.20734

20. a, b Margaret Sullivan Pepe. (2003). The Statistical Evaluation of Medical Tests for Classification and Prediction Oxford University Press

21. a, b Zobair M. Younossi, Aaron B. Koenig, Dinan Abdelatif, Yousef Fazel, Linda Henry, Mark Wymer. (2016). Global epidemiology of nonalcoholic fatty liver disease-Metaanalytic assessment of prevalence, incidence, and outcomes Hepatology, vol. 64 (1), 73-84. doi:10.1002/hep.28431

22. a, b Anna M. Diehl, Christopher Day. (2017). Cause, Pathogenesis, and Treatment of Nonalcoholic Steatohepatitis N EnglJ Med, vol. 377 (21), 2063-2072. doi:10.1056/nejmra1503519

23. ^Siddharth Singh, Alina M. Allen, Zhen Wang, Larry J. Prokop, Mohammad H. Murad, Rohit Loomba. (2015). Fibrosis Progression in Nonalcoholic Fatty Liver vs Nonalcoholic Steatohepatitis: A Systematic Review and Meta-analysis of Paired-Biopsy Studies Clinical Gastroenterology and Hepatology, vol. 13(4), 643-654.e9. doi:10.1016/j.cgh.2014.04.014

24. ^Mark CC Cheah, ArthurJMcCullough, George Boon-Bee Goh. (2017). Current Modalities of Fibrosis Assessment in Non-alcoholic Fatty Liver Disease doi:10.14218/jcth.2017.00009

25. ^Ferruccio Bonino, Umberto Arena, Maurizia R Brunetto, Barbara Coco, Mirella Fraquelli, Filippo Oliveri. (2010). Liver stiffness, a non-invasive marker of liver disease: a core study group report Antivir Ther, vol. 15 (Supp/3), 69-78. doi:10.3851/imp1626

26. ^Rohit P. Ojha, Brooke R. MacDonald, Tzu-Chun Chu, Sander Greenland. (2017). Different Cutpoints for Transient Elastography Lead to Different Associations With Cirrhosis Clinical Gastroenterology and Hepatology doi:10.1016/j.cgh.2017.11.032

27. a, b Umberto Arena, Monica Lupsor Platon, Cristina Stasi, Stefania Moscarella, Ali Assarat, Giorgio Bedogni. (2013). Liver stiffness is influenced by a standardized meal in patients with chronic hepatitis C virus at different stages of fibrotic evolution Hepatology, vol. 58 (1), 65-72. doi:10.1002/hep.26343 